\title{
ANALYSIS AND DESIGN OF CROSS-COUPLED, FOLDED E-PLANE FILTERS WITH ASYMMETRIC RESPONSES
}

\author{
Erdem Ofli, Ruediger Vahldieck, and Smain Amari ${ }^{*}$ \\ Swiss Federal Institute of Technology, IFH, Gloriastrasse 35, CH-8092, Zurich, Switzerland \\ Tel: +41-1-632 78 26, Fax: +41-1-632 11 98, E-mail: ofli@ifh.ee.ethz.ch \\ * Dept. ECE, Royal Military College of Canada, Kingston, ON, K7K 7B4, Canada
}

\begin{abstract}
Cross-coupled folded metal insert filters with asymmetric responses are introduced. The selectivity of these filters is further improved by exploiting the source-load coupling to generate up to $n$ finite transmission zeros for a filter of order $n$. Two $4^{\text {th }}$ order filters with 2 and 5 finite transmission zeros, respectively are presented to demonstrate the significantly improved response of these filters over their conventional counter part.
\end{abstract}

\section{INTRODUCTION}

Filters and duplexers at millimeter-wave frequencies require accurate theoretical design and precise fabrication to avoid the difficult task of physical fine tuning. In addition, they must be low-cost, mass producible and satisfy relatively tight electrical specifications. Modern communication systems require tight electrical specifications such as sharp cutoff between the channels to avoid cross-talk. To reduce the inherent losses in filtering structures, it is important to use the smallest number of resonators without jeopardizing the performance of the filter. It is well known that specifications for duplexer applications are more expediently met by using filters with asymmetric responses [1]. A simple mechanism to enforce the asymmetry of a response it to design a filter which is capable of generating asymmetrically prescribed finite transmission zeros.

Finite transmission zeros are most commonly generated by adding bypass of cross couplings between non-adjacent resonators. The position of the finite transmission zeros are, unfortunately, strongly sensitive to the values of the cross couplings, especially for canonical folded structures where one coefficient can affect markedly the stopband. To deal with this situation, it is of prime importance to use structures which can be designed and manufactured with very high precision. A structure which fits these specifications is the conventional straight metal-insert E-plane filter which is folded to generate finite transmission zeros. The cross-couplings can be made by etching the openings into the separation wall with high precision. The dimensions are computed accurately by using the Mode Matching Technique, therefore manual fine tuning is considerably reduced and possibly eliminated. The resulting filters are appropriate for mass-production and have significantly improved stopband performance.

In this paper, we introduce cross-coupled, folded E-plane filters with asymmetrically prescribed transmission zeros and investigate the effect of source-load coupling on their performance.

\section{FILTER CONFIGURATION AND NUMERICAL PROCEDURE}

The proposed structure of a cross-coupled folded E-plane filter with source-load coupling is shown in Figure 1. The conventional E-plane filter is folded to allow for cross-couplings. In the case of Figure 1, the structure has four cavity resonators which are directly coupled through evanescent waveguide sections (metal septa) and through a wall opening (between second and third resonators). 
Cross couplings between the first and the fourth resonators and between the source and the load are realised by introducing openings in the separating wall.

An important issue in cross-coupled filters is the realization of negative coupling using only magnetic coupling. Consider the 4-resonator filter shown in Figure 1. To generate two transmission zeros, the coupling between resonators 1 and 4 must be negative [2]. If only the fundamental resonance $\left(\mathrm{TE}_{101}\right)$ is used, the cross coupling would be positive thereby placing the transmission zeros on the real axis in the complex s-plane. To get a negative coupling, we use the next resonance, i.e. $\left(\mathrm{TE}_{102}\right)$, in resonator 3 . The coupling slot between resonators 2 and 3 is located in the second half of resonator 3 to force a negative coupling between 1 and 4 . This shifting technique is well described in [3]. On the other hand, the positive source-load coupling is achieved through an opening in the separation wall between the source and the load. However, the position of the opening must not be too close to the first coupling septum in order to get coupling with the right sign. The signs and magnitudes of the coupling coefficients can be determined from the synthesis technique developed by Bell [4]. Furthermore, the utilization of source-load coupling was also reported by Zaki and co-workers [5] and others [6]. But up to now this technique has never been applied to E-plane metal insert filters.

The Mode-Matching Technique (MMT) is used for the analysis of these structures. Since all of the openings in the geometry extend over the full height of the waveguide and the other areas of the structure have no variations in $\mathrm{y}$-direction (see Figure 1), only $\mathrm{TE}_{\mathrm{mo}}$-modes are used in the analysis. The individual discontinuities are H-plane bifurcations when viewed along the $\mathrm{z}$-axis. The overall structure is divided into general key building blocks as shown in Figure 2.

The generalized scattering matrices of all discontinuities are calculated using standard Mode Matching Technique [7,8]. By cascading the matrices appropriately, the scattering parameters of the overall structure are obtained. Since the final optimized structure may be asymmetric, all modes are considered in the analysis.

\section{DESIGN AND RESULTS}

Two different cross-coupling configurations are combined in one structure to realize a crosscoupled E-plane filter with source-load coupling (Figure 1). The structure contains two openings in the separation wall between the source and the load, and between the first and fourth resonators. As it can be seen from the response of the filter (Figure 3), 5 finite transmission zeros are obtained with this configuration. The additional zero is presumably due to the frequency-dependent coupling sections has been investigated in the literature [9]. A comparison of the asymmetric response of this filter with that of a cross-coupled filter without source-load coupling demonstrates the improved stopband of the new filter (see Figure 3). The positions of the transmission zeros are very sensitive to the small changes in the dimensions of the slots. However, these can be manufactured accurately for these filters and do not pose a serious problem.

Another advantage of these filters is their scalability. For example, to design a filter in the Ka-band, we simply scale the filter in Figure 3 and use it as a good starting point in an optimization-based design procedure. The response of the resulting filter is shown in Figure 4.

The last example is a cross-coupled bandpass filter with asymmetrically placed finite transmission zeros. Only resonators 1 and 3 are physically coupled through a slot with a positive coupling coefficient (Figure 5 (a)). The structure is analysed using both MMT and the Finite Element method (HFSS). The MMT results are shown as solid line whereas the dashed line in the same figure shows the results obtained from HFSS (Figure 5 (b)). Both simulations are in good agreement. The attenuation pole in the lower stopband is presumably due to the coupling between resonators 1 and 
4 through higher order modes. The distance between coupling septa and the opening, and the length of the septa affects the strength of the coupling. On the other hand, the pole in the upper passband of the filter is achieved by the cross coupling between resonators 1 and 3 .

\section{CONCLUSIONS}

Cross-coupled E-plane metal insert filters with asymmetric responses were introduced. The inclusion of source-load coupling in these filters further improves the stopband performance by generating additional zeros. The high precision by which these filters can be manufactured reduces and possibly eliminates the need for manual tuning thereby making them suitable for mass production.

\section{REFERENCES}

[1] R. J. Cameron, "General coupling matrix synthesis for Chebychev filtering functions", IEEE Trans. Microwave Theory Tech., vol. MTT-47, pp. 433-442, April 1999.

[2] A. E. Williams, "A four-cavity elliptic waveguide filter", IEEE Trans. Microwave Theory Tech., vol. MTT-18, pp. 1109-1114, Dec. 1970.

[3] U. Rosenberg, "New planar waveguide cavity elliptic function filters", Proc. $25^{\text {th }}$ EuMC, pp. 524-527, Sept. 1995.

[4] H. C. Bell Jr., "Canonical lowpass prototype network for symmetric coupled-resonator bandpass filters", Elec. Lett., vol.10, no. 13, pp. 265-266, June 1974.

[5] K. A. Zaki, C. Chen and A. Atia, "Modeling of coupling by probes in dual mode cavities", IEEE MTT-S Int. Symp. Dig., New York 1988, pp. 515-518.

[6] P. Roeschmann, "Compact YIG bandpass filter with finite pole frequencies for applications in microwave integrated circuits", IEEE Trans. Microwave Theory Tech., vol. MTT-21, pp. 52-57, Jan. 1973.

[7] J. Uher, J. Bornemann, and U. Rosenberg, Waveguide Components for Antenna Feed Systems: Theory and CAD, Norwood, MA: Artech House, 1993.

[8] R. Vahldieck, J. Bornemann, F. Arndt, and D. Grauerholz, "Optimized waveguide E-plane metal insert filters for millimeter wave applications", IEEE Trans. Microwave Theory Tech., vol. MTT-31, pp. 65-69, Jan. 1983.

[9] S. Amari, J. Bornemann, "Using frequency-dependent coupling to generate finite attenuation poles in direct-coupled resonator bandpass filters", IEEE Microwave and Guided Lett., pp. 404-406, Oct. 1999.
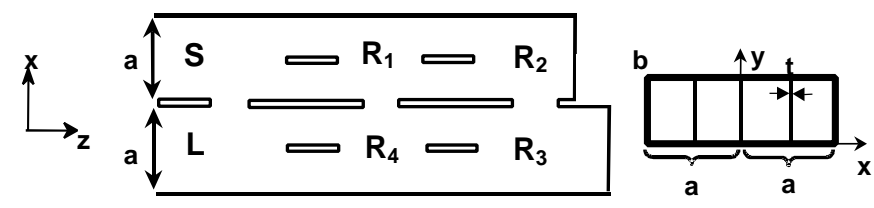

Fig. 1. Cross-coupled folded E-plane metal insert filter structure with source-load coupling (4 resonators).

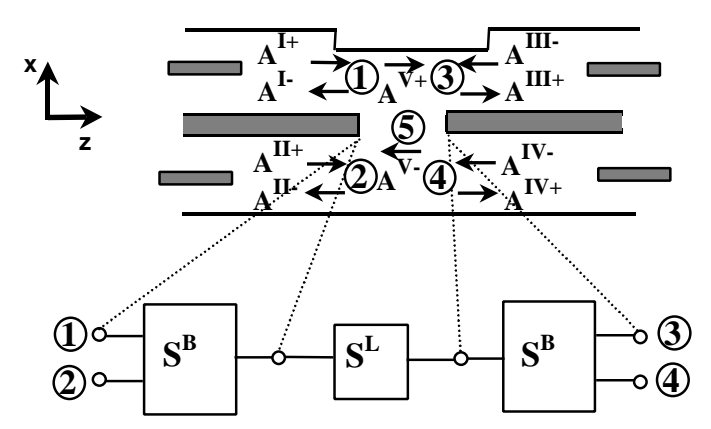

Fig. 2. General key building blocks (Waveguide bifurcations and homogeneous waveguide section). 


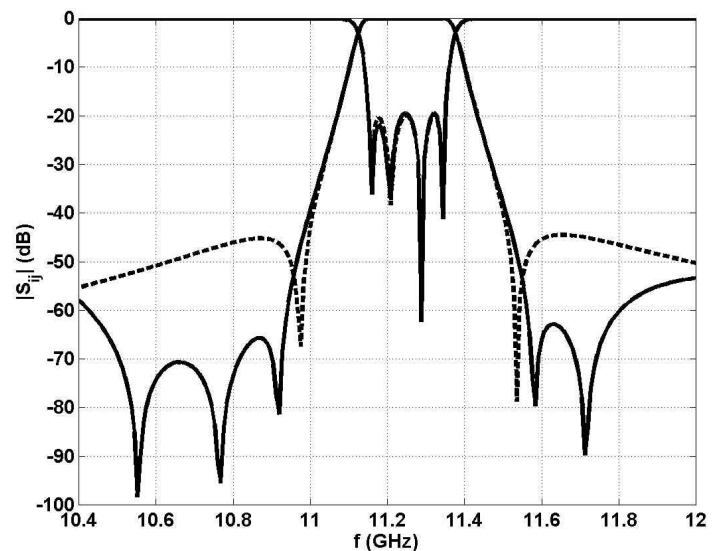

Fig. 3. Response of cross-coupled folded E-plane single metal insert filter with source-load coupling (solid line) and without source-load coupling (dashed line).
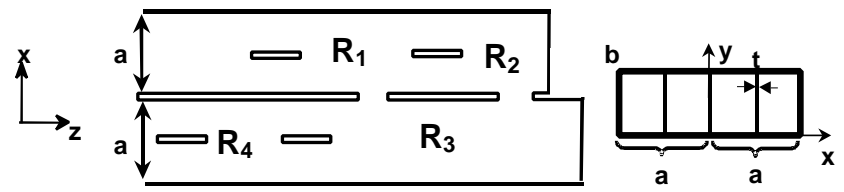

(a)

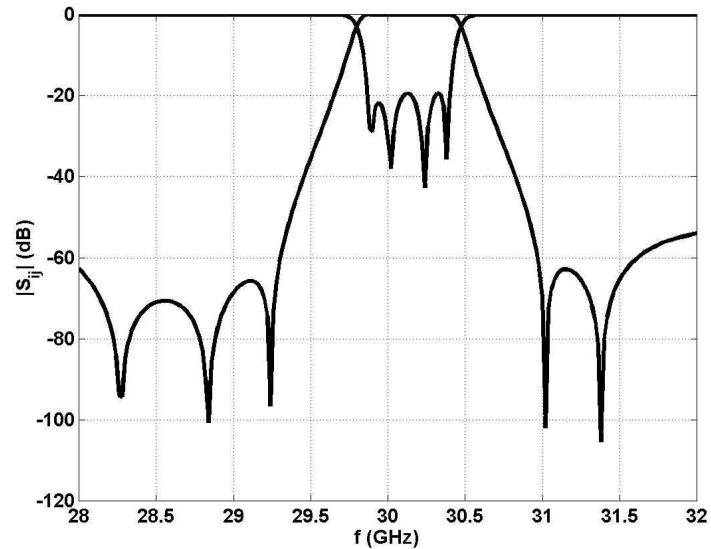

Fig. 4. Response of cross-coupled folded E-plane single metal insert filter with source-load coupling in Ka-band.

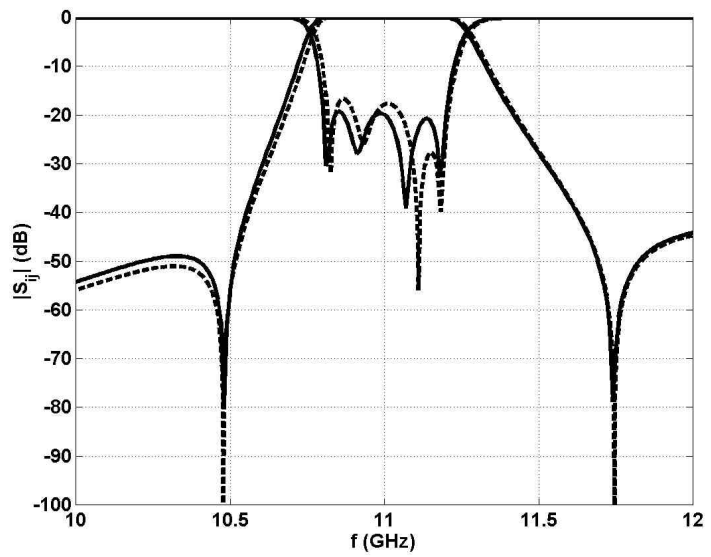

(b)

Fig. 5. (a) Structure of cross-coupled folded E-plane single metal insert filter with 4 resonators (cross coupling between $1^{\text {st }}$ and $3^{\text {rd }}$ resonators), (b) response of the filter by MMT (solid line) and HFSS (dashed line). 